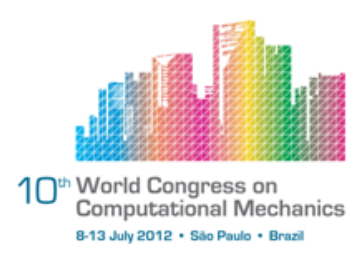

\title{
Atomistic potential based cohesive modeling for surface separation
}

\author{
Kegui $\mathrm{Xin}^{1,2}$, Minghua $\mathrm{He}^{1,2}$ \\ ${ }^{1}$ Department of Civil Engineering, Tsinghua University, Beijing, 100084, P.R. China \\ ${ }^{2}$ Key Laboratory on Civil Engineering Safety and Durability of China's Educational Ministry, \\ Beijing, 100084, P.R. China
}

\begin{abstract}
An atomistic potential based (AP-based) cohesive modeling methodology is briefly presented. For this purpose, pair potentials and and multi-body potential such as EAM are considered and their softening characteristics are shown. Based on the AP-based hyperelasicity with softening mechanism, the cohesive law in terms of cohesive traction and separation displacement is obtained. The presented method is feasible and a few remarks are given.
\end{abstract}

Keywords: cohesive law, atomistic potential, softening, hyperelasticity, continuum.

\section{INTRODUCTION}

Mechanical deformation and failure of structure and material could be classified as inherently intrinsic multiscale behavior in which the observed macroscopic material behavior is dominated by the procedure of different spatial and temporal scales (Curtin and Miller ${ }^{[1]}$ ). Continuum mechanics performs poor at the atomistic scale. Atomistic model is viewed as a precise model that could describe complex material behavior. However, the application of atomistic model for engineering problems has been restricted by its insuperable computational complexity as well as the limitation of the existing computing capacity and algorithm.

Though immature, multiscale approach as a possible solution that could combines both the advantages of continuum and atomistic methods is in the ascendant. Molecular Dynamics (including Car-Parrinello ${ }^{[2]} \mathrm{MD}$ and Born-Oppenheimer MD) is one of the most important models for atomistic simulation. Kohlhoffa et al (1991) ${ }^{[3]}$ proposed the first MD-FEM coupled method with the boundary stress compatibility conditions. After that, a lot of MD-continuum coupled methods had been given in literature. Though the MD-Continuum coupled methods possess a lot of advantages, they still are beset by several difficulties including existing of ghost forces, efficiency restriction imposed by the MD part etc.

Cauchy-Born rule was firstly seen in Cauchy's derivation of linear elastic modulus from atomistic potentials (Stakgold $(1950)^{[4]}$ ). Born and Huang (1954) ${ }^{[5]}$ systematically investigated the local homogenization kinematics theory and presented the modern form of Cauchy-Born rule. Based on Cauchy-Born rule, Tadmor and Ortiz (1996) ${ }^{[6]}$ established Quasicontinuum theory. Dupuy and Tadmor $(2005)^{[7]}$ reformed the Quasi-continuum such that the 
embedded lattice in the FEM mesh is eliminated. Besides, Coarse-Grained MD by Rudd and Broughton (1998) ${ }^{[8]}$, Virtual Internal Bond theory by Gao and Klein (1998) ${ }^{[9]}$ Bridge Scale Decomposition method by Wagner and Liu (2003) ${ }^{[10]}$ etc. are also important contributions. Xiang and Cui et al (2011) ${ }^{[11]}$ presented a nonlocal atomistic based continuum model in which high order deformation gradient tensors are considered.

Cohesive laws that describes the evolution relationship between cohesive traction and separation displacement are vital for cohesive modeling ${ }^{[12]}$. By assuming that the formation of new solid surfaces (i.e. crack etc.) is driven by the evolution of atomistic lattice, a generalized atomistic potential based (AP-based) cohesive modeling method is established.

\section{AP-based Energy Density Function}

To utilize the Atomistic Pontentials (AP), a lattice structure is needed for calculating the system energy. In physics, the lattice could be decomposed as simple Bravais lattice and complex lattice formed by multi simple Bravais lattices. A simple Bravais lattice is expressed as

$$
\tilde{\mathrm{L}}_{\mathrm{a}}=\left\{\mathbf{X}_{\mathrm{a}(\hat{I})} \mid \mathbf{X}_{\mathrm{a}(\hat{I})}=\sum a^{i} \mathbf{e}_{i}^{\mathrm{a}}+\mathbf{O}^{\mathrm{a}}, \quad a^{i} \in \mathrm{Z}^{+}\right\}
$$

where $\left\{\mathbf{e}_{i}^{\text {a }}\right\}_{i=1,2, \cdot, d}$ are the Bravais base vectors (see Weinan and Ming (2007) ${ }^{[13]}$ ). For complex lattice, the expression is given as a combination of multiple simple Bravais lattices:

$$
\mathrm{L}_{\mathrm{a}}^{*}=\left\{\tilde{\mathrm{L}}_{\mathrm{a}}+\mathbf{p}_{1}\right\} \bigcup\left\{\tilde{\mathrm{L}}_{\mathrm{a}}+\mathbf{p}_{2}\right\} \cdots \bigcup\left\{\tilde{\mathrm{L}}_{\mathrm{a}}+\mathbf{p}_{k}\right\}
$$

where $\mathbf{p}_{k}$ is the translation vector, and $k$ is an integer.

The notion of dilative open set $\Omega_{m}$ is introduced (see Friesecke et al (2008) ${ }^{[14]}$ ): Given an arbitrary open set $\Omega_{0} \in \mathrm{R}^{d}$, where $d=1,2,3$ and $m>0$, then the dilative open set $\Omega_{m}$ is given as:

$$
\Omega_{m}=\left\{m \mathbf{X} \mid \forall \mathbf{X} \in \Omega_{0}, m>0\right\}
$$

Then the finite lattice $\mathrm{L}_{\mathrm{a}}$ is given as:

$$
\mathrm{L}_{\mathrm{a}}=\mathrm{L}_{\mathrm{a}}(m)=\mathrm{L}_{\mathrm{a}}^{*} \bigcap \Omega_{m}
$$

Let us establish a lattice $\mathrm{L}_{\mathrm{a}}(m)$ made of $n_{\text {atom }}$ atoms for calculating the energy density function $W_{\mathrm{AP}}$. Let's denote the boundary the a open set $\Omega$ as $\partial \Omega$. Likewise, the lattice boundary of $\mathrm{L}_{\mathrm{a}}(m)$ is denoted as $\partial \mathrm{L}_{\mathrm{a}}(m)$. The deformation mapping $\phi_{\mathrm{L}_{\mathrm{a}}}$ for a given lattice is expressed as follow

$$
\mathbf{x}_{\mathrm{a}_{(\hat{I})}}=\phi_{\mathrm{L}_{\mathrm{a}}}\left(\mathbf{X}_{\mathrm{a}_{(\hat{I})}}, t\right)
$$

Apply an given deformation gradient $\mathbf{F}$ in terms of displacement boundary on the lattice, then the deformation of the internal part $\mathrm{L}_{\mathrm{a}}-\partial \mathrm{L}_{\mathrm{a}}$ is subject to the same deformation based on classical Cauchy-Born rule:

$$
\left.\begin{array}{l}
\forall \mathbf{X}_{\mathrm{a}(\hat{I})} \in \partial \mathrm{L}_{\mathrm{a}} \\
\mathbf{x}_{\mathrm{a}(\hat{I})}-\mathbf{o}^{\mathrm{a}}=\mathbf{F}\left(\mathbf{X}_{\mathrm{a}(\hat{I})}-\mathbf{O}^{\mathrm{a}}\right)
\end{array}\right\} \Rightarrow \mathbf{x}_{\mathrm{a}(\hat{I})}-\mathbf{o}^{\mathrm{a}}=\mathbf{F}\left(\mathbf{X}_{\mathrm{a}(\hat{I})}-\mathbf{O}^{\mathrm{a}}\right), \mathbf{X}_{\mathrm{a}(\hat{I})} \in \mathrm{L}_{\mathrm{a}}
$$


where $\mathbf{X}_{\mathrm{a}(\hat{I})}$ and $\mathbf{x}_{\mathrm{a}(\hat{I})}$ are the Material coordinates and Eulerian coordinates of the lattice $\mathrm{L}_{\mathrm{a}}(m)$. Likewise, the $\mathbf{O}^{\mathrm{a}}$ and $\mathbf{o}^{\mathrm{a}}$ denote the the material coordinates and Eulerian coordinates of the reference atom $\mathrm{a}(\mathrm{O})$ in $\mathrm{L}_{\mathrm{a}}$.

Invoking Cauchy-Born rule, the AP-based energy density function $W_{\mathrm{AP}}$ is expressed as follow:

$$
\begin{aligned}
W_{\mathrm{AP}}(\mathbf{F}) & =\lim _{m \rightarrow \infty} \frac{\sum_{\mathrm{a}(\hat{I})} E_{\mathrm{a}(\hat{I})}}{\left|\Omega_{m}\right|} \\
& =\lim _{m \rightarrow \infty} \frac{\sum_{\mathbf{x}_{\mathrm{a}(\hat{I})}, \mathbf{x}_{\mathrm{a}(\hat{j})} \in \mathbf{F L}_{\mathrm{a}}} \sum_{\hat{I} \neq \hat{j}} \frac{1}{k} \phi_{\mathrm{AP}}^{(k)}\left(\mathbf{x}_{\mathrm{a}(\hat{I})}, \cdots, \mathbf{x}_{\mathrm{a}(\hat{j})}, \cdots\right)}{\left|\Omega_{m}\right|}
\end{aligned}
$$

where $\left|\Omega_{m}\right|$ is the volume of $\Omega_{m} ; \mathbf{F L}_{\mathrm{a}}$ represents the deformed lattice under deformation $\mathbf{F}$;

Let's denote the volume of any given atom a $(\hat{I})$ on the initial configuration as $V_{0}(\mathrm{a}(\hat{I}))$, then the respective energy density function $W_{\mathrm{AP}}^{\mathrm{a}(\hat{I})}$ is given as:

$$
W_{\mathrm{AP}}^{\mathrm{a}(\hat{I})}=\frac{E_{\mathrm{a}(\hat{I})}}{V_{0}(\mathrm{a}(\hat{I}))}
$$

The volume of the finite lattice $\mathrm{L}_{\mathrm{a}}$ could be obtained as:

$$
\left|\Omega_{m}\right|=\sum_{\mathrm{a}(\hat{I}) \in \mathrm{L}_{\mathrm{a}}(m)} V_{0}(\mathrm{a}(\hat{I}))
$$

Then equation (7) is rewriten as:

$$
\begin{aligned}
W_{\mathrm{AP}}(\mathbf{F}) & =\lim _{m \rightarrow \infty} \frac{\sum_{\mathrm{a}(\hat{I}) \in \mathrm{L}_{\mathrm{a}}(m)} V_{0}(\mathrm{a}(\hat{I})) W_{\mathrm{AP}}^{\mathrm{a}(\hat{I})}}{\sum_{\mathrm{a}(\hat{I}) \in \mathrm{L}_{\mathrm{a}}(m)} V_{0}(\mathrm{a}(\hat{I}))} \\
& =\lim _{m \rightarrow \infty} \frac{\sum_{\mathrm{a}(\hat{I}) \in \mathrm{L}_{\mathrm{a}}(m)} V_{0}(\mathrm{a}(\hat{I})) W_{\mathrm{AP}}^{\mathrm{a}(\hat{I})}}{\left|\Omega_{m}\right|}=\left\langle W_{\mathrm{AP}}^{\mathrm{a}(\hat{I})}\right\rangle_{\mathrm{a}(\hat{I}) \in \mathrm{L}_{\mathrm{a}}(m)}
\end{aligned}
$$

where $\langle\cdot\rangle$ is a averaging operator. For any given finite lattice $\mathrm{L}_{\mathrm{a}}$, the respective $W_{\mathrm{AP}}$ is the total contribution of each $W_{\mathrm{AP}}^{\mathrm{a}(\hat{I})}$ for a single atom.

\subsection{Hyperelasticity for Continuum and Direct Coupling}

For the path independent hyperelasticity model of continuum (i.e. Green Elasicity, see Belytschko et al (2000) ${ }^{[15]}$ ), the respective potential functional $\Pi$ could be expressed on the initial configuration $\Omega_{0}$ with repect to the displacement field $\mathbf{u}$ :

$$
\Pi(\mathbf{u})=\int_{\Omega_{0}}\left(W_{\mathrm{e}}\left(\frac{\partial \mathbf{u}}{\partial \mathbf{X}}\right)-\mathbf{f}^{\mathrm{ext}}(\mathbf{X}) \cdot \mathbf{u}(\mathbf{X})\right) \mathrm{d} \Omega_{0}
$$

where $W_{\mathrm{e}}$ is the elastic strain energy density. For the general case of finite deformation, the symmetric second Piola-Kirchhoff stress tensor $\mathbf{S}$ and Green strain tensor $\mathbf{E}$ has the following relation:

$$
\mathbf{S}=\frac{\partial W_{\mathrm{e}}(\mathbf{E})}{\partial \mathbf{E}}=2 \frac{\partial \psi_{\mathrm{e}}(\mathbf{C})}{\partial \mathbf{C}}
$$


where $\psi_{\mathrm{e}}(\mathbf{C})$ is the deformation potential with respect to $\mathbf{C}$ and $\mathbf{C}$ is the right Cauchy-Green deformation tensor.

The hyperelasticity based on Cauchy-born rule could be derived by directly coupling $W_{\mathrm{AP}}$ and $W_{\mathrm{e}}$ :

$$
W_{\mathrm{e}}:=W_{\mathrm{AP}}(\mathrm{E})
$$

By substituting equation (10) into equation (13), the second Piola-Kirchhoff stress tensor $\mathbf{S}_{\mathrm{AP}}$ for $\Omega_{m}$ is given as:

$$
\mathbf{S}_{\mathrm{AP}}=\frac{\partial W_{\mathrm{AP}}}{\partial \mathbf{E}}=\frac{\sum_{\mathrm{a}(\hat{I}) \in \mathrm{L}_{\mathrm{a}}(m)} V_{0}(\mathrm{a}(\hat{I})) \frac{\partial W_{\mathrm{AP}}^{\mathrm{a}(\hat{I})}}{\partial \mathbf{E}}}{\sum_{\mathrm{a}(\hat{I}) \in \mathrm{L}_{\mathrm{a}}(m)} V_{0}(\mathrm{a}(\hat{I}))}
$$

The second Piola-Kirchhoff stress tensor $\mathbf{S}_{\mathrm{a}(\hat{I})}$ at the position of atom a $(\hat{(I)})$ is given as:

$$
\mathbf{S}_{\mathrm{a}(\hat{I})}=\frac{\partial W_{\mathrm{AP}}^{\mathrm{a}(\hat{I})}}{\partial \mathbf{E}}
$$

The expression for lattice $\mathrm{L}_{\mathrm{a}}(m)$ is simplified as:

$$
\mathbf{S}_{\mathrm{AP}}=\frac{\partial W_{\mathrm{AP}}}{\partial \mathbf{E}}=\frac{\sum_{\mathrm{a}(\hat{I}) \in \mathrm{L}_{\mathrm{a}}(m)} V_{0}(\mathrm{a}(\hat{I})) \mathbf{S}_{\mathrm{a}(\hat{I})}}{\sum_{\mathrm{a}(\hat{I}) \in \mathrm{L}_{\mathrm{a}}(m)} V_{0}(\mathrm{a}(\hat{I}))}=\left\langle\mathbf{S}_{\mathrm{a}(\hat{I})}\right\rangle_{\mathrm{a}(\hat{I}) \in \mathrm{L}_{\mathrm{a}}(m)}
$$

\subsection{General Form of Pair Potential based Hyperelasticity}

Let's denote pair potential as $\phi_{\mathrm{AP}}$. For lattice $\mathrm{L}_{\mathrm{a}}$, the energy density of a single atom a $(\hat{I})$ could be obtained as:

$$
\left\{\begin{array}{l}
E_{\mathrm{a}(\hat{I})}=\frac{1}{2} \sum_{\mathrm{a}(\hat{J}) \in \mathrm{L}_{\mathrm{a}} \backslash \mathrm{a}(\hat{I})} \phi_{\mathrm{AP}}\left(r_{\mathrm{a}(\hat{I}) \mathrm{a}(\hat{J})}\right) \\
W_{\mathrm{AP}}^{\mathrm{a}(\hat{I})}=\frac{1}{2 V_{0}(\mathrm{a}(\hat{I}))} \sum_{\mathrm{a}(\hat{J}) \in \mathrm{L}_{\mathrm{a}} \backslash \mathrm{a}(\hat{I})} \phi_{\mathrm{AP}}\left(r_{\mathrm{a}(\hat{I}) \mathrm{a}(\hat{J})}\right)
\end{array}\right.
$$

where $r_{\mathrm{a}(\hat{I}) \mathrm{a}(\hat{J})}=\left|\mathbf{r}_{\mathrm{a}(\hat{I}) \mathrm{a}(\hat{J})}\right|$ is the distance between atom a $(\hat{I})$ and $\mathrm{a}(\hat{J}) ; V_{0}(\mathrm{a}(\hat{I}))$ is the initial volume occupied by atom a $(\hat{I})$ on initial configuration. Then the volume $V_{0}\left(\mathrm{~L}_{\mathrm{a}}\right)$ of $\mathrm{L}_{\mathrm{a}}$ is given as:

$$
V_{0}\left(\mathrm{~L}_{\mathrm{a}}\right)=\sum_{\mathrm{a}(\hat{I})} V_{0}\left(\mathrm{a}_{\hat{I}}\right)=\sum_{\hat{I}=1}^{n_{\text {atom }}} V_{0}\left(\mathrm{a}_{\hat{I}}\right)
$$

So the energy density function $W_{\mathrm{AP}}$ of lattice $\mathrm{L}_{\mathrm{a}}$ is given as:

$$
W_{\mathrm{AP}}=\left\langle W_{\mathrm{AP}}^{\mathrm{a}(\hat{I})}\right\rangle_{\mathrm{a}(\hat{I}) \in \mathrm{L}_{\mathrm{a}}}=\frac{1}{2 V_{0}\left(\mathrm{~L}_{\mathrm{a}}\right)} \sum_{\hat{I}=1}^{n_{\text {atom }}} \sum_{\mathrm{a}(\hat{J}) \in \mathrm{L}_{\mathrm{a}} \backslash \mathrm{a}(\hat{I})} \phi_{\mathrm{AP}}\left(r_{\mathrm{a}(\hat{I}) \mathrm{a}(\hat{J})}\right)
$$

For pair potential, the second Piola-Kirchhoff stress tensor $\mathbf{S}_{\mathrm{a}(\hat{I})}$ is given as

$$
\mathbf{S}_{\mathrm{a}(\hat{I})}=\frac{1}{2 V_{0}(\mathrm{a}(\hat{I}))} \sum_{\mathrm{a}(\hat{J}) \in \mathrm{L}_{\mathrm{a}} \backslash \mathrm{a}(\hat{I})} \phi_{\mathrm{AP}}^{\prime}\left(r_{\mathrm{a}(\hat{I}) \mathrm{a}(\hat{J})}\right) \frac{\mathbf{R}_{\mathrm{a}(\hat{I}) \mathrm{a}(\hat{J})} \otimes \mathbf{R}_{\mathrm{a}(\hat{I}) \mathrm{a}(\hat{J})}}{r_{\mathrm{a}(\hat{I}) \mathrm{a}(\hat{J})}}
$$




\subsection{Multi Body Potential and EAH}

EAM is a typical multi-body potential for calculating the energy density $W_{\mathrm{a}(\hat{I})}^{\mathrm{EAM}}$ for atom a $(\hat{I})$ :

$$
W_{\mathrm{a}(\hat{I})}^{\mathrm{EAM}}=\frac{1}{V_{0}(\mathrm{a}(\hat{I}))}\left\{\digamma_{\alpha}\left(\vartheta_{\mathrm{a}(\hat{I})}\right)+\sum_{\mathrm{a}(\hat{J}) \in \mathrm{L}_{\mathrm{a} \backslash \mathrm{a}(\hat{I})}} \frac{1}{2} \phi_{\mathrm{AP}}\left(r_{\mathrm{a}(\hat{I}) \mathrm{a}(\hat{J})}\right)\right\}
$$

By substituting $W_{\mathrm{a}(\hat{I})}^{\mathrm{EAM}}$ into equation (16), EAH Embedded atom hyperelasticity model in terms of the second Piola-Kirchhoff stress tensor could be obatained (see He et al $(2012)^{[16]}$ ).

\section{Softening Check for AP-based Hyperelasticity}

Hyperelasitcity theory is important in fracture mechanics. Volokh $(2007)^{[17]}$ pointed out that the traditional hyperelastic model has a defect: as the deformation increases (in terms of right Cauchy-Green deformaton tensor $\mathbf{C}$ ), the respective deformation energy density $\psi_{\mathrm{e}}(\mathbf{C})$ could approach to infinity:

$$
\|\mathbf{C}\| \rightarrow \infty \Rightarrow \psi_{\mathrm{e}}(\mathbf{C}) \rightarrow \infty
$$

where $\|\mathbf{C}\|$ is the tensor norm for deformation tensor $\mathbf{C}$. It is not true for real material. Volokh (2007) ${ }^{[17]}$ named the traditional hyperelasticity as intact hyperelasticity and softening hyperelasticity with upper limit $\psi^{*}$ is defined as:

$$
\|\mathbf{C}\| \rightarrow \infty \Rightarrow \psi_{\mathrm{e}}(\mathbf{C}) \rightarrow \psi^{*}=\text { materialproperties }
$$

It is easy to prove the existence of an upper limit $\psi_{\mathrm{LJ}}^{*}$ for Lennard-Jones potential by adding a positive $D_{\mathrm{AP}}$ to it:

$$
\psi_{\mathrm{LJ}}^{*}=\lim _{\|\mathbf{C}\| \rightarrow \infty} W_{\mathrm{AP}}=\frac{1}{2 V_{0}\left(\mathrm{~L}_{\mathrm{a}}\right)} \sum_{\hat{I}=1}^{n_{\text {atom }}} \sum_{\mathrm{a}(\hat{J}) \in \mathrm{L}_{\mathrm{a}} \backslash \mathrm{a}(\hat{I})} D_{\mathrm{AP}}
$$

Likewise, the deformation energy density is determined by EAM such that $\psi_{\mathrm{EAM}}=$ $W_{\text {EAM. }}$. And $W_{\text {EAM }}$ could by decomposed as $W_{\mathrm{AP}}$ and $W_{\digamma_{\alpha}}$ with respect to embedded energy:

$$
W_{\mathrm{EAH}}=W_{\mathrm{AP}}+W_{\digamma_{\alpha}}
$$

where $W_{\digamma_{\alpha}}$ is contributed by embedded atom energy. For the local support properties of $\varpi_{\mathrm{a}(\hat{J})}$

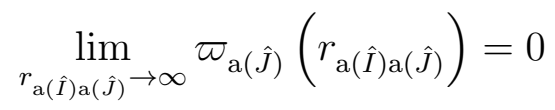

and $r_{\mathrm{a}(\hat{I}) \mathrm{a}(\hat{J})}=R_{\mathrm{a}(\hat{I}) \mathrm{a}(\hat{J})}\|\mathbf{C}\|$

$$
\lim _{\|\mathbf{C}\| \rightarrow \infty} \varpi_{\mathrm{a}(\hat{J})}\left(r_{\mathrm{a}(\hat{I}) \mathrm{a}(\hat{J})}\right)=0
$$

When $\|\mathbf{C}\| \rightarrow \infty, \vartheta_{\mathrm{a}(\hat{I})} \rightarrow 0$. For any given $\digamma_{\alpha}$, the following relation is satisfied:

$$
\lim _{\|\mathbf{C}\| \rightarrow \infty} \digamma_{\alpha} \rightarrow 0
$$

Then the softening characteristics of the estabilsed EAH is found which makes it more suitable for fracture analysis and cohesive modelling. 


\section{AP-based Cohesive Law}

In order to establish the cohesive law based on atomistic potentials, the separation displacement in terms of atomistic separation could be considered. Let's denote the distance to the first neighbour atom as $R_{0}$, then the normal separation and tangential separation representing cracking are denoted as $u_{\mathrm{n}}$ and $u_{\mathrm{t}}$. Take EAH as example, the respective cohesive law is obtained.

Take a EAM for demonstration. The chosen EAM is given as follow (Holian et al $\left.(1995)^{[18]}\right)$ :

$$
\left\{\begin{array}{l}
E_{\mathrm{a}(\hat{I})}=\frac{\chi}{2} \sum_{\mathrm{a}(\hat{J}) \in \mathrm{L}_{\mathrm{a}} / \mathrm{a}(\hat{I})} \phi_{\mathrm{AP}}+(1-\chi) \digamma_{\alpha}\left(\vartheta_{\mathrm{a}(\hat{I})}\right) \\
\digamma_{\alpha}\left(\vartheta_{\mathrm{a}(\hat{I})}\right)=\frac{d(d+1)}{2} \frac{D_{\mathrm{AP}}}{\exp (1)} \vartheta_{\mathrm{a}(\hat{I})} \ln \vartheta_{\mathrm{a}(\hat{I})}
\end{array}\right.
$$

where $\chi$ is the weight of the modified pair potential $\phi_{\mathrm{AP}}$, ranges from 0 to $1 ; d=2,3$ stands for the dimension; $D_{\mathrm{AP}}$ is the depth of the energy well; the backgound energy density $\vartheta_{\mathrm{a}(\hat{I})}$ at the position of atom a $(\hat{I})$ could be obtained by summing up the $\varpi_{\mathrm{a}(\hat{J})}$ of the surrounding atoms $\mathrm{a}(\hat{J})$ :

$$
\varpi_{\mathrm{a}(\hat{J})}\left(r_{\mathrm{a}(\hat{I}) \mathrm{a}(\hat{J})}\right)= \begin{cases}\frac{\exp (-1)}{d(d+1)}\left(\frac{r_{\max }^{2}-r_{\mathrm{a}(\hat{I}) \mathrm{a}(\hat{J})}^{2}}{r_{\max }^{2}-1}\right)^{2} & 0<r_{\mathrm{a}(\hat{I}) \mathrm{a}(\hat{J})}<r_{\max } \\ 0 & r_{\max }<r_{\mathrm{a}(\hat{I}) \mathrm{a}(\hat{J})}\end{cases}
$$

\subsection{Normal cohesvie law w.r.t EAH}

Apply normal separation in terms of deformation gradient $\mathbf{F}$ on the lattice $\mathrm{L}_{\mathrm{a}}$ :

$$
\mathbf{F}\left(u_{\mathrm{n}}\right)=\mathbf{I}+\left(\frac{u_{\mathrm{n}}}{R_{0}}\right) \mathbf{e}_{1} \otimes \mathbf{e}_{1}^{0}
$$

where $\mathbf{e}_{1}$ is the base vector on the current configuration and $\mathbf{e}_{1}^{0}$ is the respective base vector on referential configuration. Then th Green strain tensor $\mathbf{E}\left(u_{\mathrm{n}}\right)$ caused by normal separation $u_{\mathrm{n}}$ is given as:

$$
\mathbf{E}\left(u_{\mathrm{n}}\right)=\left(\left(\frac{u_{\mathrm{n}}}{R_{0}}\right)^{2}+2 \frac{u_{\mathrm{n}}}{R_{0}}\right) \mathbf{e}_{1}^{0} \otimes \mathbf{e}_{1}^{0}
$$

Then the normal cohesive law with respect to the atomistic potential is given:

$$
T_{\mathrm{n}}^{\mathrm{coh}}\left(u_{\mathrm{n}}\right)=\mathbf{S}\left(\mathbf{E}\left(u_{\mathrm{n}}\right)\right):\left(\mathbf{e}_{1}^{0} \otimes \mathbf{e}_{1}^{0}\right)
$$

where $\mathbf{e}_{1}$ denotes the normal direction for the separation. The obtained normal cohesive law is shown in Fig. 1.

\subsection{Tangential cohesvie law w.r.t EAH}

Apply tangential separation in terms of deformation gradient $\mathbf{F}$ on the lattice $\mathrm{L}_{\mathrm{a}}$ :

$$
\mathbf{F}\left(u_{\mathrm{n}}\right)=\mathbf{I}+\left(1+\frac{u_{\mathrm{t}}}{R_{0}}\right) \mathbf{e}_{1} \otimes \mathbf{e}_{2}^{0}
$$




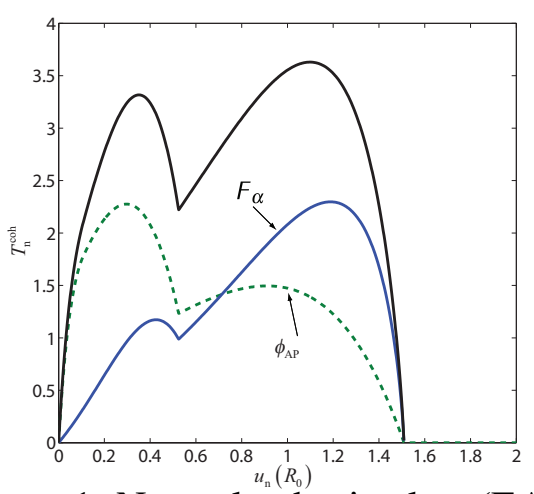

Figure 1. Normal cohesive law (EAH).

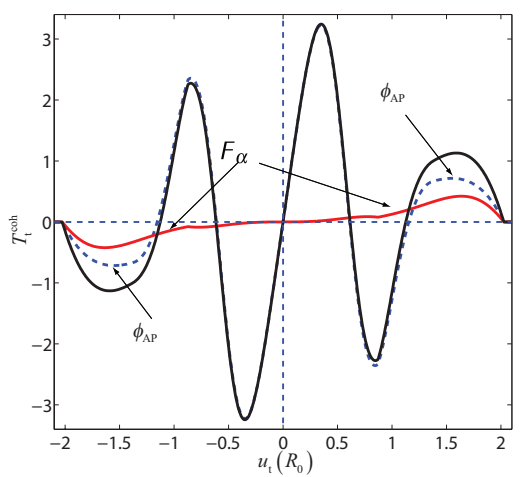

Figure 2. Tangential cohesive law (EAH).

where $\mathbf{e}_{1}$ and $\mathbf{e}_{2}$ denote the normal and tangential directions. The Green strain tensor $\mathbf{E}\left(u_{\mathrm{t}}\right)$ caused by normal separation displacement $u_{\mathrm{t}}$ is given as:

$$
\mathbf{E}\left(u_{\mathrm{t}}\right)=\left(\frac{u_{\mathrm{n}}}{R_{0}}\right)^{2} \mathbf{e}_{2}^{0} \otimes \mathbf{e}_{2}^{0}+\frac{u_{\mathrm{n}}}{R_{0}}\left(\mathbf{e}_{1}^{0} \otimes \mathbf{e}_{2}^{0}+\mathbf{e}_{2}^{0} \otimes \mathbf{e}_{1}^{0}\right)
$$

The respective tangential cohesive with respect to EAH is expressed as:

$$
T_{\mathrm{t}}^{\mathrm{coh}}\left(u_{\mathrm{t}}\right)=\mathbf{S}\left(\mathbf{E}\left(u_{\mathrm{t}}\right)\right):\left(\mathbf{e}_{1}^{0} \otimes \mathbf{e}_{2}^{0}\right)
$$

The obtained normal cohesive law is shown in Fig. 2.

\section{Remarks}

By the brief dicussion set out above, it is concluded that atomistic potential based (AP-based) cohesive modeling is feasible. For further investigation, the accuracy for such APbased methods should be improved and validated. The AP-based cohesive modeling depends on the material or the atomistic potentials. For different material system, it is vital to evaluate their differences and explore their specific behavior. The cohesion between the surfaces in front of the propagating crack tip originates from the interaction between atoms or material particles, which makes deriving the cohesive law from APs reasonable.

\section{Acknowledgements}

The authors gratefully appreciate the support from National Natural Science Foundation of China including NSFC Key Project Grant 51038006, Grant 50878117 and NSFC - High Speed Rail Joint Project Grant U1134110 as well as Tsinghua University Initiative Scientific Research Program 20101081766. 


\section{REFERENCES}

[1] Curtin W A, Miller R E. "Atomistic/continuum coupling in computational materials science”. Modelling Simul. Mater. Sci. Eng., 2003, 11(3):R33-R68.

[2] Car R, Parrinello M. "Unified Approach for Molecular Dynamics and Density-Functional Theory”. Phys. Rev. Lett., 1985, 55(22):2471-2474.

[3] Kohlhoffa S, Gumbscha P, Fischmeistera H F. "Crack propagation in b.c.c. crystals studied with a combined finite-element and atomistic model. Philosophical Magazine A, 1991, 64(4):851-878.

[4] Stakgold I. "The Cauchy relations in a molecular theory of elasticity. Quart. Appl. Math., 1950, 8:169-186.

[5] Born M, Huang K. "Dynamical Theory of Crystal Lattices. New York, London: Oxford University Press, 1954.

[6] Tadmor E B, Ortiz M, Phillips R. "Quasicontinuum analysis of defects in solids. Philosophical Magazine A, 1996, 73(6):1529-1563.

[7] Dupuy L M, Tadmor E B, Miller R E, et al. "Finite-Temperature Quasicontinuum: Molecular Dynamics without All the Atoms". Phys. Rev. Lett., 2005, 95(6):060202.

[8] Rudd R E, Broughton J Q. "Coarse-grained molecular dynamics and the atomic limit of finite elements". Phys. Rev. B, 1998, 58(10):R5893-R5896.

[9] Gao H, Klein P. "Numerical simulation of crack growth in an isotropic solid with randomized internal cohesive bonds". J. Mech. Phys. Solids, 1998, 46(2):187-218.

[10] Wagner G J, Liu W K. "Coupling of atomistic and continuum simulations using a bridging scale decomposition”. J. Comput. Phys., 2003, 190(1):249-274.

[11] Xiang M Z, Cui J Z, Tian X. "A nonlocal continuum model based on atomistic model". Sci. Sin. - Phys, Mech and Astron, 2011, 41(3): 292-301.

[12] He M H, Xin K G. "Separation work analysis of cohesive law and a consistently coupled cohesive law". Appl. Math. Mech., 2011, 32(11): 1437-1446.

[13] Weinan E, Ming P. "CauchyCBorn Rule and the Stability of Crystalline Solids: Static Problems". Arch. Ration. Mech. An., 2007, 183(2):241-297.

[14] Friesecke G, Theil F. "Validity and Failure of the Cauchy-Born Hypothesis in a TwoDimensional Mass-Spring Lattice”. J. Nonlinear Sci., 2008, 12(5):445-478.

[15] Belytschko T, Liu W K, Moran B. "Nonlinear finite elements for continua and structures". Chichester, England: John Wiley and Sons, Ltd, 2000.

[16] He M H, Li S. "An embedded atom hyperelastic constitutive model and multiscale cohesive finite element method". Comput. Mech., 2012, 49(3):337-355.

[17] Volokh K. "Hyperelasticity with softening for modeling materials failure". J. Mech. Phys. Solids, 2007, 55(10):2237-2264.

[18] Holian B L, Ravelo R. "Fracture simulations using large-scale molecular dynamics". Phys. Rev. B, 1995, 51(17):11275-11288. 